\title{
The hidden curve behind COVID-19 outbreak: the impact of delay in treatment initiation in cancer patients and how to mitigate the additional risk of dying - the head and neck cancer model
}

\author{
Leandro L. Matos ${ }^{1}$ (1) . Carlos Henrique Q. Forster ${ }^{2}$. Gustavo N. Marta ${ }^{3}$. Gilberto Castro Junior ${ }^{4}$ John A. Ridge . \\ Daisy Hirata ${ }^{6}$. Adalberto Miranda-Filho ${ }^{7}$. Ali Hosny ${ }^{8}$ - Alvaro Sanabria ${ }^{9}$ - Vincent Gregoire ${ }^{10}$. Snehal G. Patel ${ }^{11}$. \\ Johannes J. Fagan ${ }^{12}$. Anil K. D'Cruz ${ }^{13,26}$. Lisa Licitra ${ }^{14}$. Hisham Mehanna ${ }^{15}$. Sheng-Po Hao ${ }^{16}$. Amanda Psyrri ${ }^{17}$. \\ Sandro Porceddu ${ }^{18}$. Thomas J. Galloway ${ }^{19}$. Wojciech Golusinski ${ }^{20}$. Nancy Y. Lee ${ }^{21}$. Elcio H. Shiguemori ${ }^{2}$. \\ José Elias Matieli ${ }^{6}$. Ana Paula A. C. Shiguemori ${ }^{22}$ - Letícia R. Diamantino ${ }^{23}$. Luiz Felipe Schiaveto ${ }^{2} \cdot$ Lysia Leão $^{22}$. \\ Ana F. Castro ${ }^{24}$ - André Lopes Carvalho ${ }^{7}$ Luiz Paulo Kowalski ${ }^{25}$
}

Received: 23 September 2020 / Accepted: 27 February 2021 / Published online: 11 March 2021

(c) The Author(s), under exclusive licence to Springer Nature Switzerland AG 2021

\begin{abstract}
Purpose The rapid spread of the SARS-CoV-2 pandemic around the world caused most healthcare services to turn substantial attention to treatment of these patients and also to alter the structure of healthcare systems to address an infectious disease. As a result, many cancer patients had their treatment deferred during the pandemic, increasing the time-to-treatment initiation, the number of untreated patients (which will alter the dynamics of healthcare delivery in the post-pandemic era) and increasing their risk of death. Hence, we analyzed the impact on global cancer mortality considering the decline in oncology care during the COVID-19 outbreak using head and neck cancer, a known time-dependent disease, as a model.

Methods An online practical tool capable of predicting the risk of cancer patients dying due to the COVID-19 outbreak and also useful for mitigation strategies after the peak of the pandemic has been developed, based on a mathematical model. The scenarios were estimated by information of 15 oncological services worldwide, given a perspective from the five continents and also some simulations were conducted at world demographic data.

Results The model demonstrates that the more that cancer care was maintained during the outbreak and also the more it is increased during the mitigation period, the shorter will be the recovery, lessening the additional risk of dying due to timeto-treatment initiation.

Conclusions This impact of COVID-19 pandemic on cancer patients is inevitable, but it is possible to minimize it with an effort measured by the proposed model.
\end{abstract}

Keywords COVID-19 $\cdot$ Time-to-Treatment $\cdot$ Risk Evaluation and Mitigation $\cdot$ Head and Neck Neoplasms $\cdot$ Mortality

\section{Introduction}

Head and neck squamous cell carcinoma (HNSCC) have a high growth rate and causes life-threatening symptoms. Hence, deferring treatments can adversely affect HNSCC mortality rates; it is a time-dependent disease [1-3]. When only best supportive care is employed, nearly half of patients will die of cancer progression within four months [4].

Leandro L. Matos

1.matos@fm.usp.br

Extended author information available on the last page of the article
COVID-19 is likely to affect the entire world population; however, the cancer mortality risk is higher than that anticipated from initial COVID-19-related deaths [5]. The majority of cancer centers are not receiving the usual number of referred cancer patients [6]. The recent focus on COVID-19 is endorsed by the United States Center for Diseases Control (CDC) and most national and international health organizations, including the World Health Organization (WHO). Healthcare facilities have discontinued elective care, limiting their actions to offering emergency and urgent treatments [7]. Following these recommendations, all elective admissions and procedures have been postponed [8]. Subsequently, in the next months, hospitals will redirect medical care from 
COVID-19 patients toward other ailments. A surge in oncology patients, often with more advanced stage disease, should be anticipated. For HNSCC the COVID-19 pandemic has resulted in a delay of diagnosis and an increase in the time to initiation of curative treatment, thus potentially affecting outcomes [9]. National and local oncological services should be prepared to treat higher number of patients with more advanced disease as a result of the COVID-19 outbreak.

Predictive mathematical models have been useful in understanding threats to human activities [10]. They can be used to estimate the impact of severe diseases (such as cancer) in a population, and to prepare facilities to receive an additional number of patients, which could assist in establishing strategies to minimize cancer-related risk of death.

Using HNSCC as an example, this study aimed to analyze the impact of the COVID-19 pandemic on global cancer mortality in view of the pause in cancer care during recent months. Through a mathematical model, it is possible to predict the increase in the risk of dying from cancer and to suggest mitigation strategies as the COVID-19 pandemic abates.

\section{Materials and methods}

This is a predictive mathematical model based on hypothetical scenarios and on secondary data of patients treated on HNSCC.

\section{Chosen model}

The "risk of dying" model used is based on the report of Murphy et al. [1]. According to data from the National Cancer Data Base (United States), the authors examined more than 51 thousand patients and identified 67 days for time-to-treatment initiation (TTI) as the cutoff value for rising risk of death for patients treated for HNSCC. The hazard ratio was established for overall mortality according to TTI as a continuous variable and this measurement was adjusted by many covariates that included age, gender, race, insurance status (public or private insurance), facility type (community, comprehensive community or academic), distance to facility, cancer primary site (oropharynx-tonsil; oropharynx-non-tonsil; oral tongue; larynx; hypopharynx), tumor stage (AJCC seventh edition), treatment modality (surgery alone, radiation therapy alone; chemoradiation, other), year of treatment, comorbidity score and ZIP-code (level of education and income). There are few publications addressing risk of disease progression over time in cancer patients. Despite of regional characteristics (since it is based upon North American experience), Murphy's is the largest in the literature and should be considered one of the best evaluations of the TTI as an independent risk factor for death among multi-institutional patients with HNSCC.

\section{Mathematical model construction}

For the prediction of COVID-19 outbreak impact on additional risk of dying, and also the elaboration of mitigation scenarios, a mathematical model based on the estimated hazard identified by Murphy et al. [1], was developed (as demonstrated in the Supplementary Online Appendix).

To be useful, a model must provide a means to estimate the distribution of TTI, as it is the source of hazard from the operational parameters. For this, we employ simplifying assumptions: that patients present at a constant rate seeking treatment after a delay (baseline TTI) and that they wait in a line in which the first that enters is the first that is treated. From the volumes of treatment during the several phases of the process (normal before the outbreak, reduced volume due to the outbreak, mitigation periods afterwards) the number of accumulated patients-which is also the size of the waiting list—is estimated at any given time. An algorithm estimates the waiting time of a single individual considering the different treatment volumes between the time of arrival until the time of treatment. Two approaches are then available, one through simulation of every patient that arrives in the system and computing the waiting time to build the statistics. The other method is formal by assumption of continuous variables. Both produce similar results and can be used to verify the correctness of the other. The simulation approach is employed in the online calculator because of its versatility: the hazard estimation from the TTI can be changed by simply replacing the hazard function code. If the model is similar to Figure S1 (supplementary data), where the added hazard is zero until a cutoff and linear afterwards, the model parameters can be entered in the calculator interface (cutoff, slope and y-intercept), without changing the code, resulting in an operating curve similar to Figure S2.

The sensitivity of the model to parameter variation by two different approaches is demonstrated in Table S1 (supplementary data).

\section{COVID-19 cancer impact calculator (COCIC)}

The COVID-19 cancer impact calculator (COCIC) is available free online at http://cocic-calc.com, and the output data refer to all calculations. Anyone may access, use, and share data as long as this study is cited.

The website estimates needed time to recovery after the COVID-19 pandemic, the peak and average waiting time-totreatment during COVID-19 outbreak and mitigation period based upon effort, the number of patients exposed to the risk of dying, the peak value of added risk due to increased TTI and also the baseline risk of dying due to prolonged TTI 
when the time exceeds 67 days, as previously established [1]. The calculator also estimates the number of patients (at standard circumstances added to additional volume) that should be treated in different scenarios in order to reestablish the baseline risk, up to five years. It also shows the number of patients allocated in each range of TTI and the individual risk of dying due to the delay. Four charts illustrate the results: (1) number of patients waiting for treatment over time; (2) time to initiate treatment over time; (3) risk of dying from cancer over time, considering the basal risk due to the baseline waiting time and also the added risk of dying due to the extended TTI; (4) added risk of dying and the duration of the mitigation period during the first year after attention shifts from COVID-19.

The calculator is able to fit to any other situation including other varieties of cancer or time-dependent diseases by changing the parameters according to the characteristics of each illness.

\section{Data analyses and simulations}

The COCIC was used to calculate the risk of dying for patients with HNSCC in three different scenarios: (1) simulations considering different medical care volumes during COVID-19 outbreak and mitigation period; (2) sensitivity analysis considering the estimation of the impact in different services worldwide (five in Europe-Fondazione IRCCS Istituto Nazionale Tumori di Milano, Milan, Italy; University of Birmingham, UK; Leon Berard Cancer Center, Lyon, France; National Kapodistrian University of Athens Medical School, Attikon University Hospital, Athens, Greece; Poznan University of Medical Sciences at the Greater Poland Cancer Centre, Poland; three in North America; Fox Chase Cancer Center with two different services-Surgery and Radiation Therapy, Philadelphia, USA; Memorial SloanKettering Cancer Center, New York, USA; Princess Margaret Cancer Center, Toronto, Canada; three facilities in Latin America-Instituto do Cancer do Estado de Sao Paulo/Icesp with three different services-Surgery, Clinical Oncology and Radiation Therapy, Sao Paulo, Brazil; AC Camargo Cancer Center, Sao Paulo, Brazil; Universidad de Antioquia, Medellin, Colombia; two in Asia-Tata Memorial Centre, Mumbai, India; Shin Kong Wu Ho-Su Memorial Hospital and Fu Jen Catholic University in Taiwan, Taipei, Taiwan; one in Africa-University of Cape Town, South Africa; one in Oceania-Brisbane's Princess Alexandra Hospital of the University of Queensland, Queensland, Australia); and (3) simulate the HNSCC surplus deaths worldwide based on the estimated new cases and deaths for the year of 2018 available at GLOBOCAN[11]. Those estimates were used to calculate the exposed population and their added risk of dying, in order to estimate the additional number of persons that would potentially die. We considered the model for head and neck cancer globally and also stratified by continents and human development index (HDI). Attributable fraction in the exposed population (AFE) was then calculated for this population.

Exhaustive simulations were performed, in order to establish whether the model was sensitive and also consistent with the clinical impression.

\section{Results}

\section{Demonstration of COCIC use}

Considering that an institution treats a total of 100 HNSCC patients monthly, with a 60 days' time from the diagnosis to treatment under standard circumstances. During the COVID-19 outbreak, if the same institution has reduced the number of treated patients by $50 \%$ for 90 days, and subsequently after the outbreak it increases the number of patients treated by $10 \%$ above the pre-pandemic baseline, it will take 450 days to return the patients' risk of dying to baseline.

In this scenario (scenario one-Fig. 1), 1,800 patients will be exposed to the situation and the peak TTI will be 105 days (mean of 82 days). The added average risk of dying due to prolonged TTI is $6.9 \%$ at the first year and $0.4 \%$ at the second year, without additional risk after 450 days when the facility would return to pre-pandemic operation. Twelvehundred patients will be treated over a range of 0-30 additional days (TTI will be at arrange of: 60-90 days), experiencing an additional risk of dying of $2.7 \%$. Six hundred will be treated after 30 to 60 additional days (TTI will be at the range of: 90-120 days) experiencing $9.3 \%$ additional risk of dying. To return this risk to baseline in just one year instead, the increase in medical care should be at least of $12.5 \%$ during that period. Without increasing treatment volumes (i.e., returning only to standard operational volume following the recovery from the outbreak), patients will have a steady $11.5 \%$ additional risk of dying at that facility, simply as a result of the long-lasting increase in TTI, that in this new scenario would be of 105 days.

In another scenario (scenario two-Fig. 1), a facility might have a baseline TTI higher than 67 days (i.e., beyond the threshold of the model for risk of dying), lower volume of care during the outbreak, and also lower volume expansion during mitigation period. With 120 days as baseline TTI, 100 patients treated monthly, reduction of medical volume by $70 \%$ over 90 days, and an increase of $5 \%$ in medical care volume after the pandemic, it will take 1,260 days to mitigate the additional risk of dying due to the prolonged TTI with a baseline risk of dying due to the increased TTI of $16.0 \%$. Then the peak value of TTI will be 183 days (mean of 151 days) in 4,501 patients. The added risk of dying is $16 \%$ at the first year, $11.6 \%$ at the second, $6.4 \%$ at the third 


\section{SCENARIO 1}

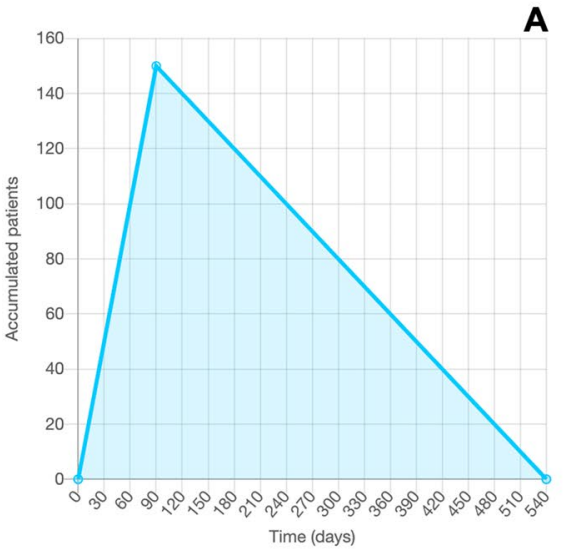

SCENARIO 2

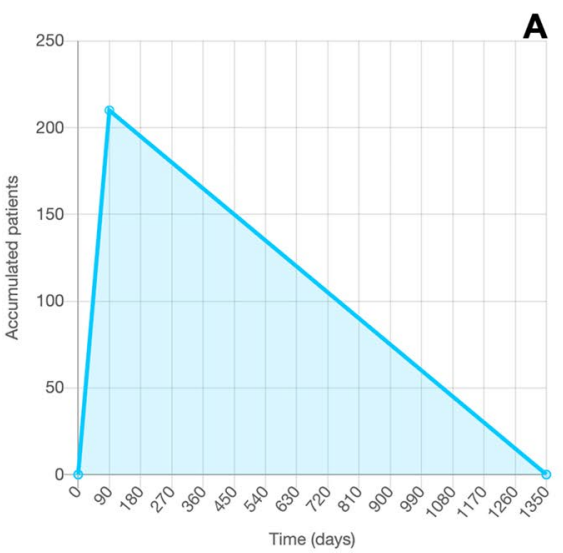

A

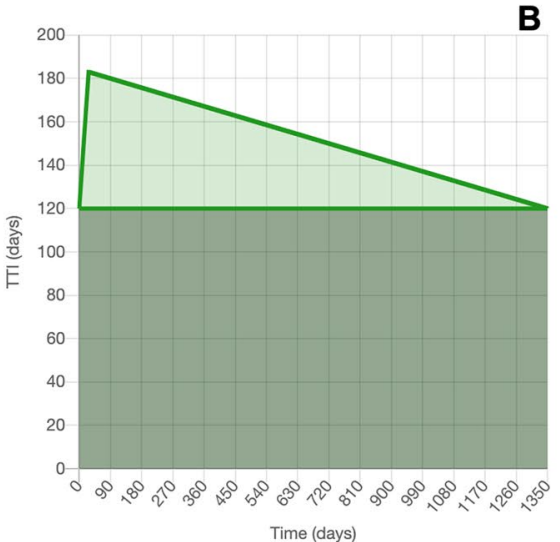

B
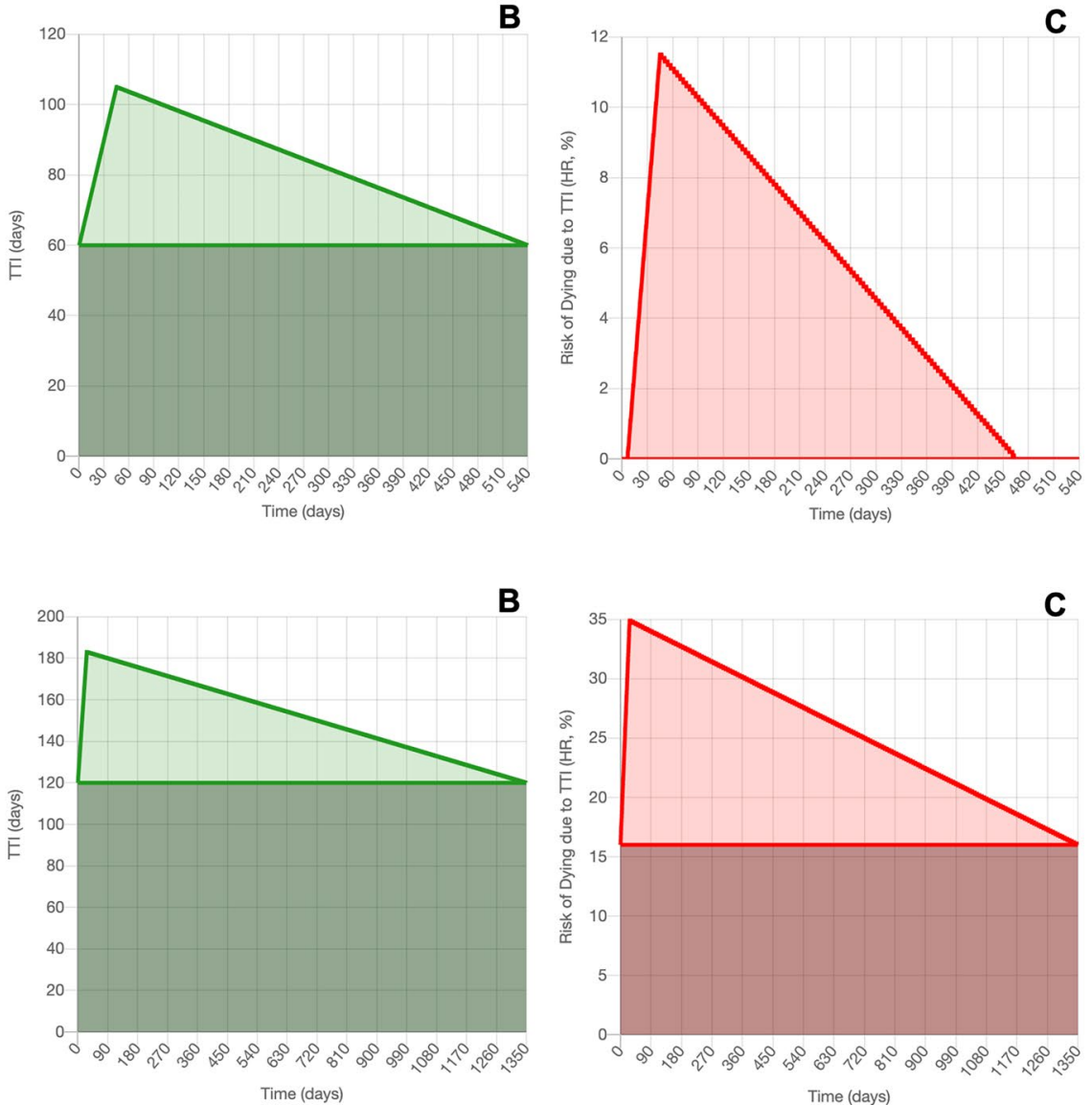

Fig. 1 Graphic representation of the model considering two different simulations. Scenario one considering a baseline time-to-treatment initiation (TTI) of 60 days in a facility that usually treat 100 patients per month. During the COVID-19 outbreak the medical care volume was reduced to $50 \%$ and the service expect an increase of $10 \%$ in medical care volume after a 90 days period of pandemic. a 150 patients waiting for treatment at the peak of the outbreak, with additional 450 days to return to the baseline condition, during the mitigation period; $\mathbf{b}$ average additional TTI of 45 days (peak of overall 105 days), during time to return to the baseline condition (TTI of 60 days); c average of $4.4 \%$ of additional risk of dying (peak of $11.5 \%$ for overall risk) during time to return to the baseline condition (no risk); Scenario two considering a baseline time-to-treat-

and $1.4 \%$ at the fourth year, without additional elevated risk after that. Under these circumstances, 2,143 patients will be treated over a range of $0-30$ additional days (120-150 days as final TTI), experiencing an additional risk of dying of $4.5 \%(20.5 \%$ as overall risk; $4.5 \%$ of additional risk $+16.0 \%$ of baseline risk), 2,143 will be treated over 30-60 additional days (TTI: $150-180$ days in total) with additional risk of $13.5 \%$ (29.5\% as overall risk) and 215 individuals will be treated over additional 60-90 days (TTI: 180-210 days in total) with $18.4 \%$ of additional risk of dying ( $34.4 \%$ as overall). To be able to mitigate these risks in just one year (then returning to the baseline condition), the increase in treatment ment initiation (TTI) of 120 days in a facility that usually treat 100 patients per month. During the COVID-19 outbreak the medical care volume was reduced to $30 \%$ and the service expect an increase of 5\% in medical care volume after a 90 days period of pandemic. a 210 patients waiting for treatment at the peak of the outbreak, with additional 1,260 days to return to the baseline condition, during the mitigation period; $\mathbf{b}$ average additional TTI of 63 days (peak of overall 183 days), during time to return to the baseline condition (TTI of 120 days); $\mathbf{c}$ average of $9.4 \%$ of additional risk of dying (peak of $37.5 \%$ for overall risk) during time to return to the baseline condition (risk of 16\%). The difference between both scenarios is not only the shape of the curves but also the axis values

volume should be at least of $17.5 \%$; an $8.8 \%$ increase will lead to a return to baseline in two years. Without mitigation efforts, the patients would experience an 18.9\% long-term additional risk of dying at that facility, representing a new baseline of $34.9 \%$ (18.9\% of additional risk plus the standard baseline on 16\%) as risk of dying from the COVID-19 pandemic's delay in treatment for HNSCC.

The more medical care is delivered during the outbreak and the greater the expansion in medical care volume during the mitigation period, the quicker will be the recovery and lower the additional risk of dying due to a longer TTI. Simulations considering duration of the outbreak, medical 
care volume during the outbreak and mitigation regimens are demonstrated in Figs. 2 and 3, considering 66 days of baseline TTI (the limit days for no baseline risk of dying due to waiting time for treatment). In general, a reduction of $50 \%-70 \%$ during 60-90 days of outbreak, results in an additional risk of dying due to longer TTI over at least two years if the medical care volume rises $10 \%$ to $20 \%$ above baseline during the mitigation period. Detailed data are shown in Table S2 and Figure S3 (supplementary data).

\section{Estimating real-life scenarios}

To better predict the local effect of COVID-19 pandemic in HNSCC practice, different centers worldwide were asked to relate their real-life expectations.

The usual TTI ranged from 21 to 150 days (mean of 50 days; standard-deviation of 36 days), with a mean medical care reduction of $41.4 \%$ during the outbreak and $16.8 \%$ volume expansion after the pandemic, with an estimated duration of 77 days of COVID-19 outbreak. The time to mitigate the additional risk of dying due to the increased TTI range from 24 to 400 days, with a 1 -year added risk of dying ranging from $1.2 \%$ to $19.8 \%$, with five services $(27.8 \%)$ showing additional risk. The greater the effort and the shorter the preexisting TTI of the medical facility, the lower time needed to return to baseline and the lower the additional risk of death due to HNSCC. These results are described in Table 1 and complete data in Tables S3 and S4 (supplementary data).
It may not prove possible for each institution to execute its proposed increase in volume as a long-term mitigation strategy. Even so, in nine facilities (50\%) patients could experience an increased risk of dying after the pandemic varying from $0.7 \%$ to $26.8 \%$ yearly due to long-standing novel TTI. Even in those centers that are unlikely to sustain any additional risk, the increment in TTI will average $45.1 \%$ (from 50 to 91 days), as shown in Table S5 (supplementary data).

Those facilities with a longer baseline TTI will be required to adopt more aggressive strategies in order to reestablish their baseline settings. This is likely to be more difficult precisely because they are located in countries with limited resources. Centers with sufficiently short pre-COVID TTI may be able to return their circumstances to baseline without sustaining increased mortality; such institutions are usually in environments with less straitened circumstances.

\section{Extrapolation of the model to demographic data}

To estimate the number of patients likely to succumb because of the decrease in medical care during COVID-19 pandemic, the added calculated risk (hazard ratio) from the model was applied to calculate the AFE using the estimate of new cases and deaths from HNSCC worldwide available at GLOBOCAN for the year of 2018 [11].

As parameters on the COCIC calculator, we considered a $40 \%$ reduction in cancer care during a 90 days outbreak, the baseline TTI was calculated using the data provided by the real-data scenarios and the literature for those continents
Fig. 2 First-year added risk of dying due to delay in time-totreatment initiation considering the reduction of medical care during four simulations of COVID-19 outbreak duration. These risks represent situations without increase in medical care volume after the pandemic

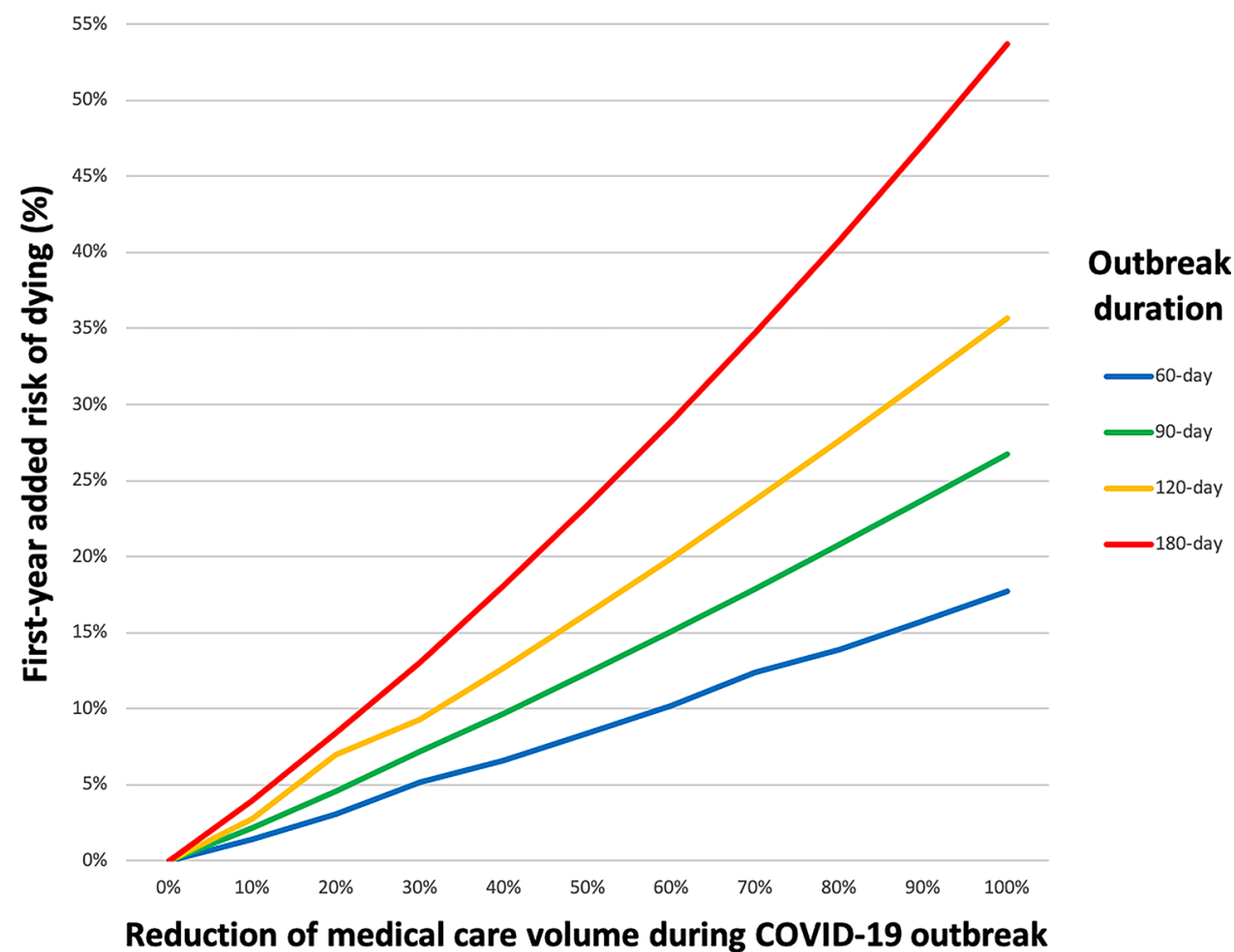


Fig. 3 First-year added risk of dying (line) and necessary time of effort to recovery (bar) due to delay in time-to-treatment initiation considering the reduction of medical care during three simulations of COVID-19 outbreak duration $(60,90$ and 120 days), and also de mitigation of these risks based on increase of medical care volume after the pandemic (increase of $5 \%, 10 \%, 15 \%, 20 \%$ and $50 \%$ ). Complete and descriptive data of all these values are shown in Table S2 and Figure S3 (supplementary data)
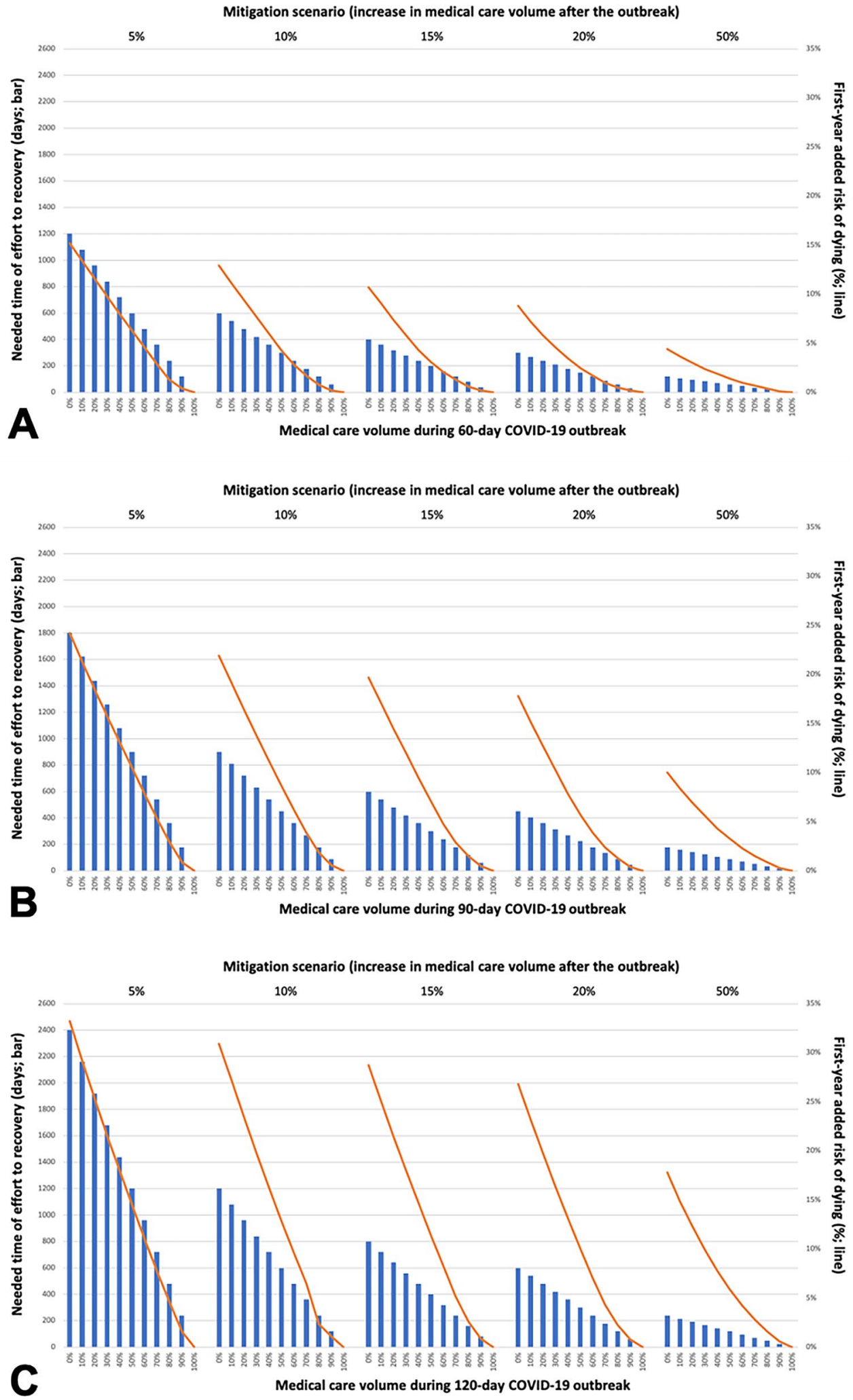

and groups of HDI countries for a better representation of TTI [12-17]. We inputted these data and simulated different efforts of mitigation, varying from $50 \%$ to no mitigation at all. In a scenario of no mitigation, the number of additional patients that could die due to HNSCC could rise from $0 \%$ to $10.8 \%$ yearly after the outbreak depending on geography and the HDI; this represents up to additional 47,558 deaths/year as a result of increased TTI due to the COVID-19 outbreak. 
Table 1 Information of different centers worldwide, regarding medical care volume before and during COVID-19 outbreak, estimations of mitigation scenarios after the outbreak and estimative of treatment impact

\begin{tabular}{|c|c|c|c|c|c|c|c|c|}
\hline $\begin{array}{l}\text { Continents } \\
\text { and Coun- } \\
\text { tries }\end{array}$ & $\begin{array}{l}\text { Usual Time- } \\
\text { to-Treatment } \\
\text { Initiation }^{\mathrm{a}}\end{array}$ & $\begin{array}{l}\text { Medical care } \\
\text { reduction during } \\
\text { the outbreak }\end{array}$ & $\begin{array}{l}\text { Medical care } \\
\text { increment after } \\
\text { the outbreak }\end{array}$ & $\begin{array}{l}\text { Estimated dura- } \\
\text { tion (days) of } \\
\text { restricted opera- } \\
\text { tion due to the } \\
\text { outbreak }\end{array}$ & $\begin{array}{l}\text { Needed time of } \\
\text { effort to recov- } \\
\text { ery (days) }\end{array}$ & TTI (Peak) & $\begin{array}{l}\text { Patients } \\
\text { at risk } \\
\text { (exposed) }\end{array}$ & $\begin{array}{l}\text { Added } \\
\text { risk } \\
\text { (aver- } \\
\text { age-1st } \\
\text { year) }\end{array}$ \\
\hline \multicolumn{9}{|l|}{ Europe } \\
\hline Greece & 30 & $20.0 \%$ & $6.0 \%$ & 90 & 300 & 48 & 650 & $0 \%$ \\
\hline Italy & 30 & $42.9 \%$ & $14.3 \%$ & 120 & 360 & 81 & 2,240 & $0 \%$ \\
\hline UK & 30 & $30.0 \%$ & $0.0 \%$ & 120 & $\infty$ & 66 & 1,201 & $\mathrm{c}$ \\
\hline France & 40 & $0 \%$ & $0.0 \%$ & 0 & - & - & - & - \\
\hline Poland & 45 & $40.0 \%$ & $100.0 \%$ & 60 & 24 & 69 & 504 & $0 \%$ \\
\hline \multicolumn{9}{|l|}{ Latin America } \\
\hline Brazil & 30 & $47.8 \%$ & $20.0 \%$ & 90 & 215 & 73 & 2,339 & $0 \%$ \\
\hline Brazil & 80 & $41.7 \%$ & $16.7 \%$ & 160 & 400 & 147 & 1,121 & $13.2 \%$ \\
\hline Colombia & 81 & $50.0 \%$ & $0.0 \%$ & 90 & $\infty$ & 126 & N/E & $12.5 \%$ \\
\hline Brazil & 120 & $51.6 \%$ & $21.0 \%$ & 90 & 222 & 166 & 645 & $6.0 \%$ \\
\hline Brazil & 150 & $50.0 \%$ & $16.7 \%$ & 90 & 270 & 195 & 1,441 & $0 \%$ \\
\hline \multicolumn{9}{|c|}{ North America } \\
\hline USA & 21 & $50.0 \%$ & $17.6 \%$ & 30 & 85 & 36 & 652 & $0 \%$ \\
\hline USA & 30 & $25.0 \%$ & $20.8 \%$ & 60 & 72 & 45 & 529 & $0 \%$ \\
\hline USA & 25 & $80.0 \%$ & $50.0 \%$ & 28 & 45 & 47 & 98 & $0 \%$ \\
\hline Canada & 56 & $\mathrm{~b}$ & $20.0 \%$ & 0 & - & - & - & - \\
\hline \multicolumn{9}{|l|}{ Asia } \\
\hline Taiwan & 23 & $60.0 \%$ & $0.0 \%$ & 30 & $\infty$ & 41 & N/E & $\mathrm{c}$ \\
\hline India & 30 & $68.2 \%$ & $0.0 \%$ & 60 & $\infty$ & 71 & $\mathrm{~N} / \mathrm{E}$ & $1.2 \%$ \\
\hline Africa & & & & & & & & \\
\hline South Africa & 30 & $46.7 \%$ & $0.0 \%$ & 270 & $\infty$ & 156 & N/E & $19.8 \%$ \\
\hline \multicolumn{9}{|l|}{ Oceania } \\
\hline Australia & 47 & $0.5 \%$ & $0.0 \%$ & 0 & - & - & - & - \\
\hline
\end{tabular}

${ }^{\mathrm{a}}$ Under standard circumstances

${ }^{\mathrm{b}}$ Increase of medical care due to a particular situation

'Even though no mitigation strategy led to $0 \%$ increment in risk of dying, the long-standing 'novel TTI' of the service will be higher

TTI = time-to-treatment initiation; N/E: not estimated (absence of mitigation strategy); “-” services that do not experimented reduction of medical care volume during the outbreak

An increase of 5\% in medical care, lasting up to two years, after the outbreak could reduce this number to 40,121 cases in total, while $20 \%$ of volume increase in the services during a six months of mitigation period reflects in 13,406 additional deaths, a reduction of $67 \%$ when compared to the $5 \%$ mitigation effort. The impact of additional deaths is proportionally worse on the less developed countries and in medium and low HDI settings (Table 2).

\section{Discussion}

The outcomes of cancer patients vary among institutions and countries due to differences in varieties of cancers; prevention and screening policies; stage at diagnosis and access to adequate specialized healthcare[18, 19]. Better disease outcomes in HNSCC patients are basically related to early diagnosis, prompt treatment (ideally with a multidisciplinary approach), early rehabilitation and avoiding subsequent exposure to known risk factors such as tobacco and alcohol [20]. During COVID-19 pandemic, all these domains were affected since healthcare systems turned human and material resources into the treatment of infected patients. The North American Center for Disease Control [8] recommendations to suspend all elective healthcare and to provide only urgent and emergency treatment, were followed by numerous national and international medical organizations. In many countries, hospitals have converted operating suites into intensive care rooms due to the large proportion of patients with respiratory failure. As one of the indirect consequences, 


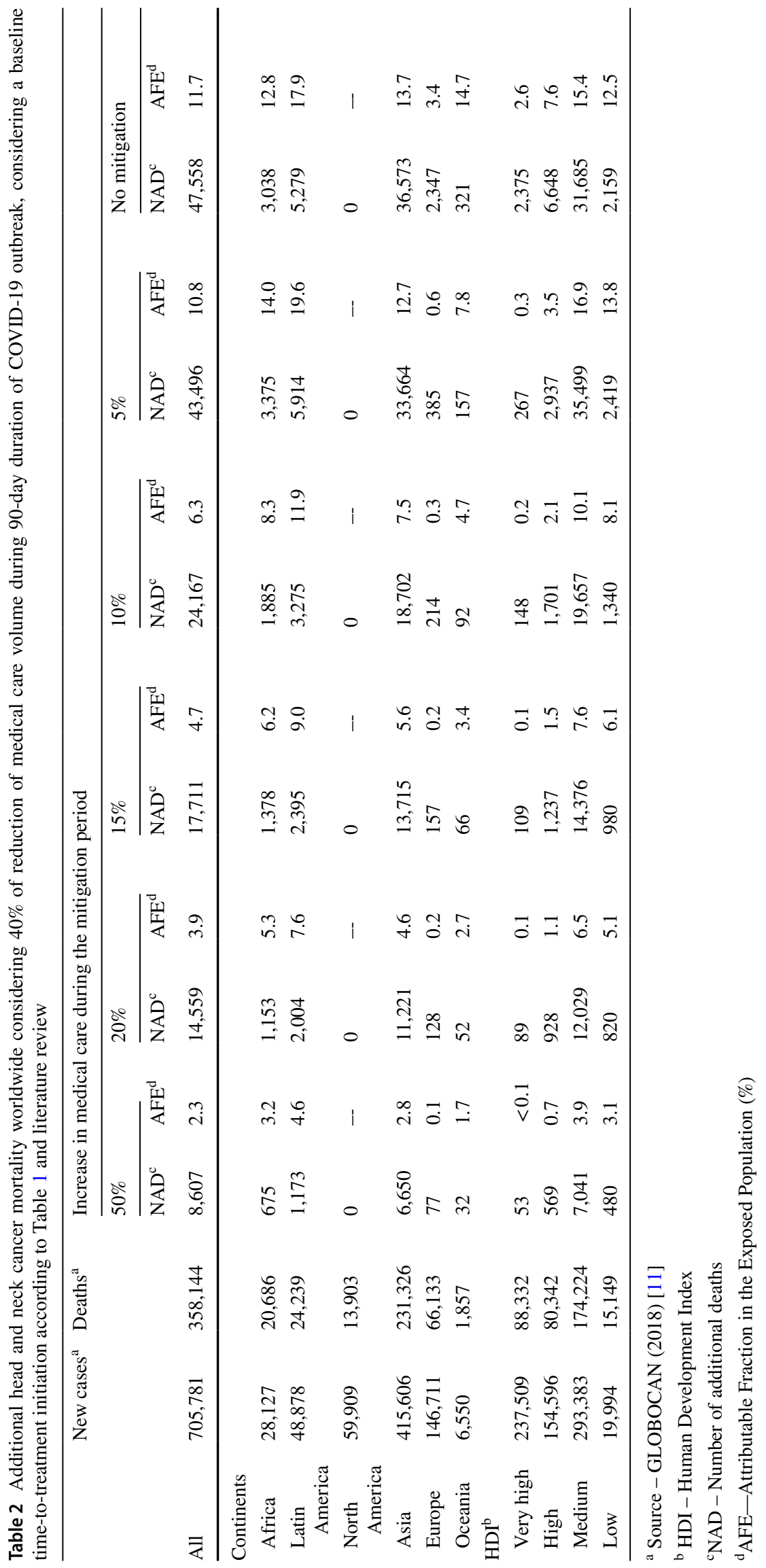


we will face an increase in the mortality ratio for chronic and non-communicable diseases. A likely increase in the risk of death or disability from stroke is expected [21]. Surprisingly, hospitalizations for acute myocardial infarction declined in northern Italy and in California, during the COVID-19 pandemic, more than would be expected on the basis of typical seasonal variation alone [22, 23].

Little organized guidance was afforded surrounding urgent systemic treatment, radiation therapy, and elective surgery for cancer patients $[5,24,25]$ There is evidence highlighting the effects of COVID-19 pandemic on cancer care. Two Chinese reports showed that the COVID-19 pandemic was associated with increased severe events and cancer-related deaths that were attributed to impediments to oncologic care [26, 27]. Lai et al. [28] analyzed the recent changes in cancer care delivery in response to COVID-19. Population-based health records in England, and also USA Surveillance, Epidemiology, and End Results (SEER) data were used to estimate an excess of 6,270 cancer deaths at 1 year in England and 33,890 in the USA. It is, therefore, necessary to reduce delays in cancer diagnosis, resume disrupted treatment, as well as to reallocate the resources diverted during the COVID-19 pandemic [29].

The understanding of the natural history of untreated cancer is essential to appreciate the effects of the COVID-19 pandemic on the survival expectations of cancer patients. The evolution of a HNSCC is rapid, from initial presentation until it becomes untreatable [30]. About 50\% of untreated individuals will die in four months, only $12 \%$ will survive more than 12 months and almost none will survive for five years $[4,31,32]$.

Treatment package time has significant prognostic impact in HNSCC patient outcomes; and timeliness of care delivery is regarded as a major quality metrics of oncologic care [1, 3, 33-36].

This study employs HNSCC as a model to estimate the impact of COVID-19 pandemic on its worldwide cancer mortality considering the reduction of medical care volume during the outbreak. The mortality of those patients with HNSCC is closely related to presenting stage and also to longer time-to-treatment initiation, both affected when attention is diverted from cancer care. Thus, HNSCC is an appropriate disease to analyze the COVID-19 pandemic in terms of excess deaths, to propose how to reduce them and also to ascertain how much time will be needed to return to normal after the expected surge in patients presenting after the pandemic.

Although the model was designed to estimate the impact on increased risk of dying due to the outbreak for head and cancer patients, if considering that HNSCC represent only around $4 \%$ of all cancer worldwide, one can extrapolate that the toll of additional cancer deaths due to the COVID-19 outbreak to be in the millions, a number way higher than the deaths due to COVID-19 (close to 400,000 in early June, 2020 and around two million people in the year of 2020), unfortunately. Nevertheless, to afford more accurate estimates the model should be adjusted to each individual clinical setting; covariates; and different TTI risk values for each cancer. Even with otherwise imprecise estimates it is evident that the number of deaths due cancer and other diseases will be substantially higher than those directly caused by COVID-19, in the aftermath, despite efforts at mitigation.

Considering the existing heterogeneity among different healthcare services around the globe in terms of the capacity for admitting newly diagnosed HNSCC patients, different treatment recommendations, diverse limitations in access to material resources and technologies, and reimbursement policies and procedures, one could expect a great variability in terms of capacity to accommodate any expansion of healthcare delivery after the pandemic period. Already frail healthcare networks will worsen due to economic recession and consequent reduction in healthcare expenditures in lowincome and middle-income countries [19, 37]. Historically, recessions contribute to increases in mortality among the more vulnerable populations, and in cancer patients especially due to COVID-19 it should not be different [38-40].

We have developed a mathematical model based on the estimated hazard identified by Murphy et al. to predict impact of the COVID-19 outbreak on additional risk of dying from HNSCC and also to propose some mitigation scenarios. The assumptions used in this study, were based on 51,655 patients from the National Cancer Data Base and indicated that 67 days was the non-zero threshold, maintained in the validation set. The mortality risk rises substantially after 67 days, after adjusting for covariates. Time to diagnosis and adequate staging, pre-treatment mortality ratio and the number of patients treated with palliative care were not considered, and consequently, overall HNSCC mortality risk may be even higher as time-to-treatment initiation increases. The mathematical model proposed here can be useful and may be adapted for other malignancies in which a time-to-treatment-initiation-dependent mortality risk exists.

As shown, a smaller decline in healthcare delivery during the outbreak and a more rapid and pronounced expansion afterwards will engender a quicker return to baseline mortality rates and reduce the additional risk of dying due to longer TTI. Our model cannot address different capacities of diverse healthcare systems or the access to treatment modalities in varied geographic areas. The financial and human resources required to increase access to radiation therapy, surgical treatment, and systemic treatment are likely to prove limiting in many regions. Our study provides data that can be used for individual institutions or entire national health systems to evaluate strategies and to prepare guidelines, infrastructure and their workforce to face the upcoming challenges. As demonstrated with the 
simulated data using the COCIC calculator, the scenario of no mitigation is the one that brings the most damage to the head and neck cancer population, it creates a novel baseline TTI that in most of the cases are followed by a long-lasting increase in additional deaths. The modeling of the data also showed that even a small increment of 5\% volume in oncology services provided can bring the additional risk to an end; moreover, it appears in most of the simulations that an effort between 10 and $20 \%$ increase in medical care during the mitigation period results in return to baseline in six to 12 months in most of the scenarios tested.

We developed an easy-to-use, accurate instrument (COCIC), capable of predicting the impact of delayed treatment of head and neck cancer patients due to the COVID-19 pandemic. The tool is free and available online and also allows estimating mitigation strategies to reduce the associated mortality in the post-outbreak era in different scenarios and different diseases. Our tool does not consider specifically some important variables for the estimating the risk of dying for cancer, especially for HNSCC, such as stage, treatment modality, tumor site, age and symptoms. Although the hazard used on risk estimation was based on the study of Murphy et al. [1] and it is controlled by multiple variables, including those and many others. Based on the principle of equity, all cancer patients waiting for treatment should have the same chance to be treated during the proposed mitigation period. However, we know that in a scenario of limited resources and very long waiting time period, a prioritization strategy should be necessary. Our group is now working on a new tool, additional to the COCIC, in order to provide a prioritization model based on some relevant variables, but we believe that the COCIC itself still an important tool to provide mitigation strategies in a generalized way.

In conclusion, the proposed model demonstrates that the more the healthcare delivery is maintained during the COVID-19 outbreak and also the more it is increased during the mitigation period, the sooner will be the recovery and smaller the additional risk of death due prolonged time to begin treatment in an already stressed system [41]. This impact of COVID-19 pandemic on cancer patients is inevitable, but it is possible to minimize it with a planned effort.

Supplementary Information The online version contains supplementary material available at https://doi.org/10.1007/s10552-021-01411-7.

Acknowledgment Study developed by Head and Neck Surgery Department of the University of São Paulo Medical School, Sao Paulo, Brazil, and Instituto Tecnológico de Aeronáutica, São José dos Campos, Brazil, in collaboration with 15 other centers Worldwide.
Funding The authors declare that this paper was elaborated in the absence of any commercial or financial relationships that could be considered as a potential conflict of interest.

Data availability The authors could provide any additional data upon request to the correspondent author.

\section{Declarations}

Conflict of interest Hisham Mehanna is the director and a shareholder of Warwickshire Head and Neck Clinic LTD, he also reports personal fees from AstraZeneca, personal fees from MSD, personal fees from Sanofi Pasteur, personal fees from Merck, grants from GSK Biologicals, grants from MSD, grants from Sanofi Pasteur, grants from AstraZeneca, non-financial support from Merck, non-financial support from MSD, all disclosures are outside the submitted work. Leandro L. Matos, Carlos Henrique Q. Forster, Gustavo N. Marta, Gilberto Castro Junior, John A. Ridge, Daisy Hirata, Adalberto Miranda-Filho, Ali Hosny, Alvaro Sanabria, Vincent Gregoire, Snehal G. Patel, Johannes J. Fagan, Anil K. D'Cruz, Lisa Licitra, Sheng-Po Hao, Amanda Psyrri, Sandro Porceddu, Thomas J. Galloway, Wojciech Golusinski, Nancy Y. Lee, Elcio H. Shiguemori, José Elias Matieli, Ana Paula A. C. Shiguemori, Letícia R. Diamantino, Luiz Felipe Schiaveto, Lysia Leão, Ana F. Castro, André Lopes Carvalho, Luiz Paulo Kowalski declare that they have no conflict of interest.

\section{References}

1. Murphy CT, Galloway TJ, Handorf EA et al (2016) Survival Impact of Increasing Time to Treatment Initiation for Patients With Head and Neck Cancer in the United States. J Clin Oncol 34:169-178

2. Xiao R, Ward MC, Yang K et al (2018) Increased pathologic upstaging with rising time to treatment initiation for head and neck cancer: A mechanism for increased mortality. Cancer 124:1400-1414

3. Yao JJ, Zhang F, Gao TS et al (2019) Survival impact of radiotherapy interruption in nasopharyngeal carcinoma in the intensitymodulated radiotherapy era: A big-data intelligence platformbased analysis. Radiother Oncol 132:178-187

4. Kowalski LP, Carvalho AL (2000) Natural history of untreated head and neck cancer. Eur J Cancer 36:1032-1037

5. Hanna TP, Evans GA, Booth CM (2020) Cancer, COVID-19 and the precautionary principle: prioritizing treatment during a global pandemic. Nat Rev Clin Oncol. https://doi.org/10.1038/s4157 1-020-0362-6

6. Rosenbaum L (2020) The Untold Toll-The Pandemic's Effects on Patients without Covid-19. N Engl J Med. https://doi.org/10.1056/ NEJMms 2009984

7. MSKCC (2020) Covid Subcommittee of the O.R. Executive Committee at Memorial Sloan Kettering-Cancer Surgery and COVID19. Ann Surg Oncol 27:1713-1716

8. CDCP. (2020) Interim U.S. Guidance for Risk Assessment and Public Health Management of Healthcare Personnel with Potential Exposure in a Healthcare Setting to Patients with Coronavirus Disease (COVID-19). Centers for Disease Control and Prevention.

9. Yuen E, Fote G, Horwich P et al (2020) Head and neck cancer care in the COVID-19 pandemic: A brief update. Oral Oncol. https:// doi.org/10.1016/j.oraloncology.2020.104738

10. Altrock PM, Liu LL, Michor F (2015) The mathematics of cancer: integrating quantitative models. Nat Rev Cancer 15:730-745 
11. IARC (2020) Global Cancer Observatory (GLOBOCAN). International Agency for Research on Cancer, Lyon, France

12. Esmaelbeigi F, Hadji M, Harirchi I, Omranipour R, vand Rajabpour M, Zendehdel K. (2014) Factors affecting professional delay in diagnosis and treatment of oral cancer in Iran. Arch Iran Med $17: 253-257$

13. Iyer HS, Kohler RE, Ramogola-Masire D et al (2019) Explaining disparities in oncology health systems delays and stage at diagnosis between men and women in Botswana: A cohort study. PLoS ONE. https://doi.org/10.1371/journal.pone.0218094

14. Joshi P, Nair S, Chaturvedi P, Nair D, Agarwal JP, D'Cruz AK (2014) Delay in seeking specialized care for oral cancers: experience from a tertiary cancer center. Indian J Cancer 51:95-97

15. Coca-Pelaz A, Takes RP, Hutcheson K et al (2018) Head and Neck Cancer: A Review of the Impact of Treatment Delay on Outcome. Adv Ther 35:153-160

16. Flukes S, Garry S, Hinton-Bayre A, Lindsay A (2019) Pre-treatment wait time for head and neck cancer patients in Western Australia: description of a new metric and examination of predictive factors. ANZ J Surg 89:858-862

17. Gigliotti J, Madathil S, Makhoul N (2019) Delays in oral cavity cancer. Int J Oral Maxillofac Surg 48:1131-1137

18. de Souza JA, Hunt B, Asirwa FC, Adebamowo C, Lopes G (2016) Global Health Equity: Cancer Care Outcome Disparities in High-, Middle-, and Low-Income Countries. J Clin Oncol 34:6-13

19. Choi HCW, Lam KO, Pang HHM, Tsang SKC, Ngan RKC, Lee AWM (2019) Global comparison of cancer outcomes: standardization and correlation with healthcare expenditures. BMC Public Health 19:1065

20. Mehanna H, Paleri V, West CM, Nutting C (2010) Head and neck cancer-Part 1: Epidemiology, presentation, and prevention. BMJ. https://doi.org/10.1136/bmj.c4684

21. van der Worp B, Sandset EC, Dichgans M. (2020) Likely increase in the risk of death or disability from stroke during the covid-19 pandemic. European Stroke Organization.

22. Solomon MD, McNulty EJ, Rana JS et al (2020) The Covid-19 Pandemic and the Incidence of Acute Myocardial Infarction. N Engl J Med. https://doi.org/10.1056/NEJMc2015630

23. De Filippo O, D’Ascenzo F, Angelini F et al (2020) Reduced Rate of Hospital Admissions for ACS during Covid-19 Outbreak in Northern Italy. N Engl J Med. https://doi.org/10.1056/NEJMc 2009166

24. Chaves ALF, Castro AF, Marta GN et al (2020) Emergency changes in international guidelines on treatment for head and neck cancer patients during the COVID-19 pandemic. Oral Oncol. https ://doi.org/10.1016/j.oraloncology.2020.104734

25. Marron JM, Joffe S, Jagsi R, Spence RA, Hlubocky FJ (2020) Ethics and Resource Scarcity: ASCO Recommendations for the Oncology Community During the COVID-19 Pandemic. J Clin Oncol. https://doi.org/10.1200/JCO.20.00960

26. Liang W, Guan W, Chen R et al (2020) Cancer patients in SARSCoV-2 infection: a nationwide analysis in China. Lancet Oncol 21:335-337

27. Zhang L, Zhu F, Xie L et al (2020) Clinical characteristics of COVID-19-infected cancer patients: a retrospective case study in three hospitals within Wuhan, China. Ann Oncol. https://doi. org/10.1016/j.annonc.2020.03.296

28. Lai A, Peasea L, Banerjee A, et al. (2020) Estimating excess mortality in people with cancer and multimorbidity in the COVID-19 emergency. Research Gate.

29. Kutikov A, Weinberg DS, Edelman MJ, Horwitz EM, Uzzo RG, Fisher RI (2020) A War on Two Fronts: Cancer Care in the Time of COVID-19. Ann Intern Med. https://doi.org/10.7326/ M20-1133

30. Kowalski LP, Carvalho AL (2001) Influence of time delay and clinical upstaging in the prognosis of head and neck cancer. Oral Oncol 37:94-98

31. Shimkin MB, Griswold MH, Cutler SJ (1984) Classics in oncology. Survival in untreated and treated cancer. CA Cancer J Clin 34:282-294

32. Stell PM (1989) Survival times in end-stage head and neck cancer. Eur J Surg Oncol 15:407-410

33. Bolwell BJ, Khorana AA (2016) Enhancing Value for Patients With Cancer: Time to Treatment as a Surrogate for Integrated Cancer Care. J Natl Compr Canc Netw 14:115-116

34. Guttmann DM, Kobie J, Grover S et al (2018) National disparities in treatment package time for resected locally advanced head and neck cancer and impact on overall survival. Head Neck 40:1147-1155

35. Ho AS, Kim S, Tighiouart M et al (2018) Quantitative survival impact of composite treatment delays in head and neck cancer. Cancer 124:3154-3162

36. Committee on Quality of Health Care in America (2001) Crossing the Quality Chasm: A New Health System for the 21st Century. National academy press, Washington (DC)

37. Carvalho AL, Pintos J, Schlecht NF et al (2002) Predictive factors for diagnosis of advanced-stage squamous cell carcinoma of the head and neck. Arch Otolaryngol Head Neck Surg 128:313-318

38. Hone T, Mirelman AJ, Rasella D et al (2019) Effect of economic recession and impact of health and social protection expenditures on adult mortality: a longitudinal analysis of 5565 Brazilian municipalities. Lancet Glob Health 7:e1575-e1583

39. Kuderer NM, Choueiri TK, Shah DP et al (2020) Clinical impact of COVID-19 on patients with cancer (CCC19): a cohort study. Lancet. https://doi.org/10.1016/S0140-6736(20)31187-9

40. Lee LYW, Cazier JB, Starkey T et al (2020) COVID-19 mortality in patients with cancer on chemotherapy or other anticancer treatments: a prospective cohort study. Lancet. https://doi.org/10.1016/ S0140-6736(20)31173-9

41. Ruiz-Patino A, Arrieta O, Pino LE et al (2020) Mortality and Advanced Support Requirement for Patients With Cancer With COVID-19: A Mathematical Dynamic Model for Latin America. JCO Glob Oncol 6:752-760

Publisher's Note Springer Nature remains neutral with regard to jurisdictional claims in published maps and institutional affiliations. 


\section{Authors and Affiliations}

Leandro L. Matos ${ }^{1}\left(\mathbb{0}\right.$. Carlos Henrique Q. Forster ${ }^{2}$. Gustavo N. Marta ${ }^{3}$. Gilberto Castro Junior ${ }^{4}$. John A. Ridge ${ }^{5}$. Daisy Hirata $^{6}$ - Adalberto Miranda-Filho ${ }^{7}$. Ali Hosny ${ }^{8}$ - Alvaro Sanabria ${ }^{9}$ - Vincent Gregoire ${ }^{10}$. Snehal G. Patel ${ }^{11}$. Johannes J. Fagan ${ }^{12}$. Anil K. D'Cruz ${ }^{13,26}$. Lisa Licitra ${ }^{14}$. Hisham Mehanna ${ }^{15}$. Sheng-Po Hao ${ }^{16}$. Amanda Psyrri ${ }^{17}$. Sandro Porceddu ${ }^{18}$. Thomas J. Galloway ${ }^{19}$. Wojciech Golusinski ${ }^{20}$. Nancy Y. Lee ${ }^{21}$. Elcio H. Shiguemori ${ }^{2}$. José Elias Matieli ${ }^{6}$. Ana Paula A. C. Shiguemori ${ }^{22}$ - Letícia R. Diamantino ${ }^{23}$ - Luiz Felipe Schiaveto ${ }^{2}$. Lysia Leão ${ }^{22}$. Ana F. Castro ${ }^{24}$ - André Lopes Carvalho ${ }^{7}$ Luiz Paulo Kowalski ${ }^{25}$

Carlos Henrique Q. Forster

forster@ita.br

Gustavo N. Marta

gustavo.marta@hc.fm.usp.br

Gilberto Castro Junior

gilberto.castro@usp.br

John A. Ridge

john.ridge@fccc.edu

Daisy Hirata

daisy@ita.br

Adalberto Miranda-Filho mirandaa@fellows.iarc.fr

Ali Hosny

ali.hosni@rmp.uhn.ca

Alvaro Sanabria

alvarosanabria@gmail.com

Vincent Gregoire

Vincent.GREGOIRE@lyon.unicancer.fr

Snehal G. Patel

patels@mskcc.org

Johannes J. Fagan

johannes.fagan@uct.ac.za

Anil K. D'Cruz

docdcruz@gmail.com

Lisa Licitra

Lisa.Licitra@istitutotumori.mi.it

Hisham Mehanna

h.mehanna@bham.ac.uk

Sheng-Po Hao

shengpo747@gmail.com

Amanda Psyrri

psyrri@med.uoa.gr

Sandro Porceddu

Sandro.Porceddu@health.qld.gov.au

Thomas J. Galloway

Thomas.Galloway@fccc.edu

Wojciech Golusinski

wgolus@ump.edu.pl

Nancy Y. Lee

leen2@mskcc.org

Elcio H. Shiguemori

elcio@ieav.cta.br

José Elias Matieli

matieli@ita.br
Ana Paula A. C. Shiguemori

anapaula.acs@ifsp.edu.br

Letícia R. Diamantino

diamantino.leticia@gmail.com

Luiz Felipe Schiaveto

luizfschiaveto@gmail.com

Lysia Leão

lysia.leao@aluno.ifsp.edu.br

Ana F. Castro

anafmferreira@yahoo.com

André Lopes Carvalho

carvalhoa@iarc.fr

Luiz Paulo Kowalski

lp_kowalski@uol.com.br

1 Department of Head and Neck Surgery, Instituto Do Cancer Do Estado de São Paulo (ICESP), University of São Paulo Medical School, and Faculdade Israelita de Ciências da Saúde Albert Einstein Medical School, Enéas de Carvalho Aguiar avenue, 255, 8th floor, room 8174, Sao Paulo, SP 05403-000, Brazil

2 Instituto Tecnológico de Aeronáutica (ITA), São José dos Campos, Brazil

3 Division of Radiation Oncology, Department of Radiology Oncology, Instituto Do Cancer Do Estado de São Paulo (ICESP), University of São Paulo Medical School, and Department of Radiation Oncology, Hospital Sírio-Libanês, São Paulo, Brazil

4 Department of Clinical Oncology, Instituto Do Cancer Do Estado de São Paulo (ICESP), University of São Paulo Medical School, São Paulo, Brazil

5 Department of Surgical Oncology, Fox Chase Cancer Center, Philadelphia, USA

6 Instituto Tecnológico de Aeronáutica (ITA), Faculdade de Ciências Médicas de São José Dos Campos, São José dos Campos, Brazil

7 International Agency for Research On Cancer, Lyon, France

8 Department of Radiation Oncology, University of Toronto, Toronto, Canada

9 Department of Surgery, Universidad de Antioquia, Hospital Universitario San Vicente Fundacion-CEXCA Centro de Excelencia en Enfermedades de Cabeza Y Cuello, Medellin, Colombia

10 Department of Radiation Oncology, Léon Bérard Cancer Center, Lyon, France 
11 Head and Neck Surgery Service, Memorial Sloan Kettering Cancer Center, New York, NY, USA

12 Division of Otolaryngology, University of Cape Town Medical School, Cape Town, South Africa

13 Oncology Apollo Hospitals, Navi, Mumbai, India

14 Fondazione IRCCS Istituto Nazionale Dei Tumori and University of Milan, Milan, Italy

15 Institute for Head and Neck Studies and Education, University of Birmingham, Birmingham, UK

16 Department of Otolaryngology of Shin, Kong Wu Ho-Su Memorial Hospital and Fu Jen Catholic University in Taiwan, Taipei, Taiwan

17 Clinical Oncology Department, National Kapodistrian University of Athens Medical School, Attikon University Hospital, Athens, Greece

18 Department of Radiation Oncology, Brisbane's Princess Alexandra Hospital of the University of Queensland, Brisbane, QLD, Australia
19 Department of Radiation Oncology, Fox Chase Cancer Center, Philadelphia, USA

20 Department of Head and Neck Surgery, Poznan University of Medical Sciences, The Greater Poland Cancer Centre, Poznań, Poland

21 Department of Clinical Oncology, Memorial Sloan Kettering Cancer Center, New York, NY, USA

22 Instituto Federal de Educação, Ciência E Tecnologia de São Paulo, Jacareí, Brazil

23 Faculdade de Ciências Médicas de São José Dos Campos, São José dos Campos, Brazil

24 Clinical Oncology Department, Lenitudes Medical Center \& Research, Santa Maria da Feira, Portugal

25 Department of Head and Neck Surgery, University of São Paulo Medical School, and Department of Head and Neck Surgery and Otorhinolaryngology A C Camargo Cancer Center, São Paulo, Brazil

26 President-Elect, Union for International Cancer Control (UICC), Geneve, Switzerland 